\title{
Light-driven oscillations of entangled nematic colloidal chains
}

\author{
M. Gomilšsk ${ }^{1}$, D. Sečㄹ, M. Škarabott ${ }^{1,2}$, M. Ravnik ${ }^{3}$, S. Žumer ${ }^{1,2}$, and I. Muševič ${ }^{1,2, a}$ \\ 1 Jožef Stefan Institute, Jamova 39, SI-1000 Ljubljana, Slovenia \\ 2 Faculty of Mathematics and Physics, University of Ljubljana, Jadranska 19, SI-1000 Ljubljana, Slovenia \\ 3 Rudolf Peierls Centre for Theoretical Physics, University of Oxford, 1 Keble Road, Oxford 0X13NP, UK
}

Received 18 June 2010

Published online: 7 November 2010

(C) The Author(s) 2010. This article is published with open access at Springerlink.com

\begin{abstract}
Laser tweezers have been used to drive the oscillations of a chain of entangled colloidal particles in the nematic liquid crystal 5CB. The amplitude and phase of light-driven oscillations have been determined for the motion of individual colloidal particles. The collective motion of $4.8 \mu \mathrm{m}$ silica particles is highly damped for a driving frequency above $0.5 \mathrm{~Hz}$. The results were compared to an effective bead-spring model, where the motion of elastically coupled particles is hindered by viscous damping and hydrodynamic coupling. Qualitative agreement between theory and experiment was obtained.
\end{abstract}

\section{Introduction}

Nematic colloids are dispersions of solid, liquid or gaseous objects in a nematic liquid crystal. During recent years they have attracted a lot of attention [1-13] because of the unusual ability of self- or directed assembly of colloidal particles into ordered colloidal crystalline structures [13] and superstructures [14]. The assembled colloidal structures are bound by strong inter-colloidal forces, which are mediated by the elastic distortion of the nematic liquid crystals. Binding energies of several $1000 k_{B} T$ per micrometer size particles have been determined, which makes nematic colloidal assemblies very robust against external perturbations and therefore potentially interesting for technological applications in photonic devices.

When a colloidal particle is immersed in a nematic liquid crystal, the surface of the particle interacts with liquid crystalline molecules and forces them to align into a certain direction with respect to the surface. As the surfaces of colloidal particles are curved, liquid crystalline molecules cannot fill the space around the particle without elastic distortion of the nematic LC, and without creating defects. As a result, the nematic colloidal particle induces a long-range elastic distortion of the director field, describing the local orientation of the nematic liquid crystal around the inclusion and this distorted region is accompanied by a topological defect in a form of a point or closed loop. In the core of the defect, the nematic order parameter is strongly decreased with respect to the bulk value. For a perpendicular (homeotropic) anchoring of nematic LC at the surface of the microsphere, dipolar

\footnotetext{
a e-mail: igor.musevic@ijs.si
}

and quadrupolar symmetries of the colloidal particle were predicted and observed [15].

The elastic distortion caused by the surface anchoring of LC molecules at curved surfaces is of long range and is responsible for interaction forces when two colloidal particles are positioned close to each other. The reason for the appearance of forces is simple: as the regions of elastic deformation around each colloidal particle overlap, this overlapping may be energetically in favor for both particles, which results in an attractive force. If however, the deformed regions are not energetically favorable for both particles, a repulsive force between the colloidal particles is generated.

Whereas forces and force equilibria in nematic colloids are now well understood, much less is known about the dynamic properties of nematic colloids. Brownian motion of colloidal particles in the nematic LC is usually used to measure the viscosity coefficients, which are needed to calculate the separation dependence of pair forces and binding potentials in nematic colloids. It is known that the motion of micrometer-size objects in the nematic LC is highly damped due to the large viscosity of the nematic LC. Now, as we are able to assemble relatively large-size nematic colloidal chains and $2 \mathrm{D}$ crystals of very different types, interesting questions arise about the collective dynamic motion of such assemblies. Such a motion can in principle be determined in two different ways: i) by analyzing the thermal noise spectra of the assemblies, or ii) by applying an external force and measuring the response of the structure. We have chosen the second option and have used the laser tweezers [16-21] to apply the timeharmonic external force to a 1D colloidal assembly. Using video-microscopy and particle tracking technique, we 
a)

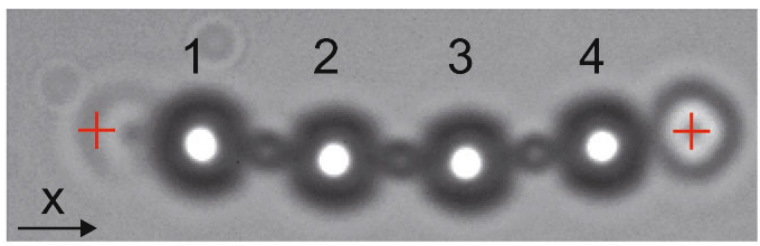

b)

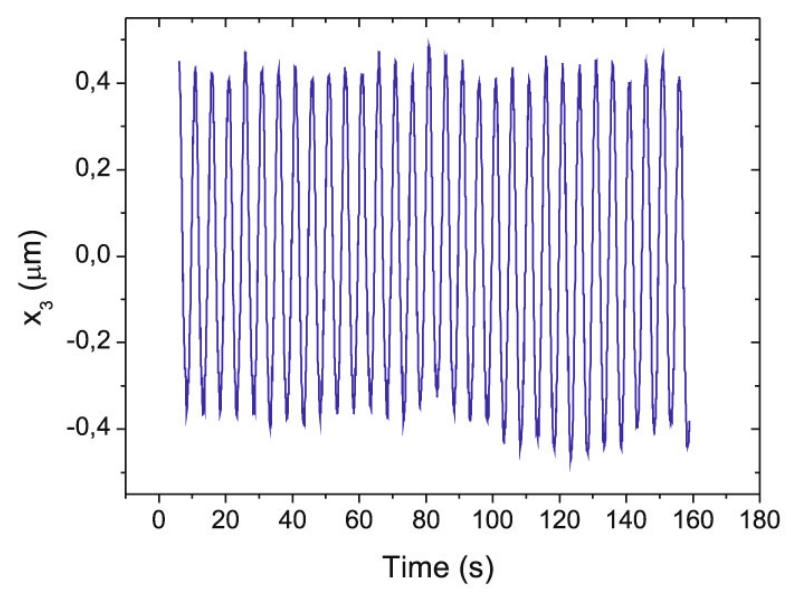

Fig. 1. (a) Entangled hyperbolic defect colloidal chain, stretched by two laser tweezers. The left laser trap at colloid 1 is fixed, while the right laser trap at colloid 4 is harmonically oscillated with fixed amplitude and frequency. (b) Recorded horizontal positions of the third particle $x_{3}$, while the right laser tweezer was oscillating with frequency of $0.2 \mathrm{~Hz}$ and amplitude of $0.75 \mu \mathrm{m}$.

have measured the motion of individual colloidal particles in the colloidal chain and compared it to the theoretical calculations.

\section{Experiment and results}

In the experiment we have measured driven oscillations of the entangled hyperbolic defect colloidal chain of silica microspheres (fig. 1a), where the time-periodic external force was provided by the strongly focused light of the laser tweezers, driving small oscillations of the captured colloidal particle.

Entangled nematic colloidal chains were reported recently by our group [22]. Colloidal particles in the entangled colloidal chain are bound by topological defect lines in a form of closed loops, which are entangling an arbitrary number of particles. There are three known types of entangled nematic colloidal chains and we have here studied the entangled hyperbolic point defect colloidal chain (see fig. 4b), where a single and non-twisted defect loop encircles all colloidal particles and there is a hyperbolic point defect between each pair of colloidal particles [22].

The dispersion of silica spheres with diameter of $4.8 \mu \mathrm{m}$ (Bangs Laboratories) in NLC pentylcyanobiphenyl (5CB) was prepared first. The dispersion was inserted into the planar nematic cell with thickness of $6-7 \mu \mathrm{m}$, made of two glass substrates covered with the rubbed polyimide alignment layer to induce uniform planar alignment of the NLC throughout the cell. The surfaces of colloidal particles were treated chemically to induce perpendicular alignment of LC molecules at their surface. In a thick planar cell such particles are accompanied with the hyperbolic hedgehog defect, whereas in a thin planar cell, this point defect opens into the "Saturn ring" defect loop. Since in our experiment the thickness of the cell is only $\sim 50 \%$ bigger than the diameter of the particles, almost all particles are encircled with the "Saturn ring" defect loop [23]. We have used an optical tweezers setup to assemble colloidal chains. The tweezers setup is based on two acoustooptic deflectors, driven by a computerized system (Aresis, Tweez 70) and a cw argon laser (Coherent, Innova 90C) at $514 \mathrm{~nm}$ was used as a laser source. Using the laser tweezers as a tool to manipulate nematic colloidal particles, we have assembled these Saturn-like particles into entangled hyperbolic defect colloidal chains of $N$ particles. These chains are spontaneously oriented in a direction perpendicular to the rubbing direction.

Once the entangled colloidal chains were assembled, two optical traps of the laser tweezers were used in the driven-oscillation experiment. The first optical trap of the laser tweezers was positioned at the left-end particle of the colloidal chain, whereas the second optical trap of the same optical power was positioned at the right-end particle in the colloidal chain. The focus of the right laser tweezers trap was then set into a time-periodic motion in a direction $x$ along the chain, with a pre-selected frequency and amplitude. The oscillations of each particle in the colloidal chain were observed by the optical microscopy using an inverted polarizing microscope (Nikon Eclipse, TE2000-U) with water immersion microscope objective (Nikon, NIR Apo 60/1.0W). The positions of individual particles were video monitored and determined by the off-line analysis of captured frames with an accuracy of $\pm 5 \mathrm{~nm}$. This unusually high spatial resolution in determining the absolute position of a colloidal particle is based on a rather simple but efficient method [24]. Images of micrometer-sized colloidal particles are taken during their motion using a good quality optical microscope and a digital camera. Then, an off-line analysis of particle's positions is performed for each recorded frame. In this analysis, we are seeking for the best overlap of the image of the colloidal particle with a graphic object of the same shape (i.e. filled circle) and the colloidal image. A typical accuracy for determining colloidal position of several nanometers could be achieved using the software employing image recognition algorithms to track the bead [25].

We focused on driven-oscillation experiments with $N=4$ colloidal particles in the entangled hyperbolic point defect colloidal chain. The fourth colloidal particle (see fig. 1a) was driven into harmonic oscillations with an amplitude of $0.75 \mu \mathrm{m}$ and frequency which was varied in different experiments from $0.05 \mathrm{~Hz}$ to $2.5 \mathrm{~Hz}$. We observed that at higher frequencies the colloidal particles were not oscillating any more due to the high viscosity of the liquid crystal. Positions of all four particles were monitored with the rate of $50 \mathrm{fps}$, and total acquisition time was around 
a)

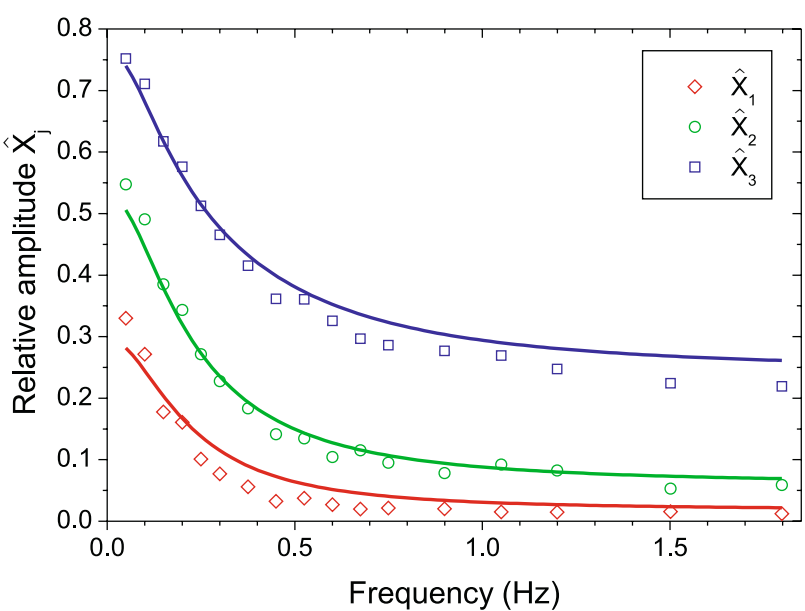

b)

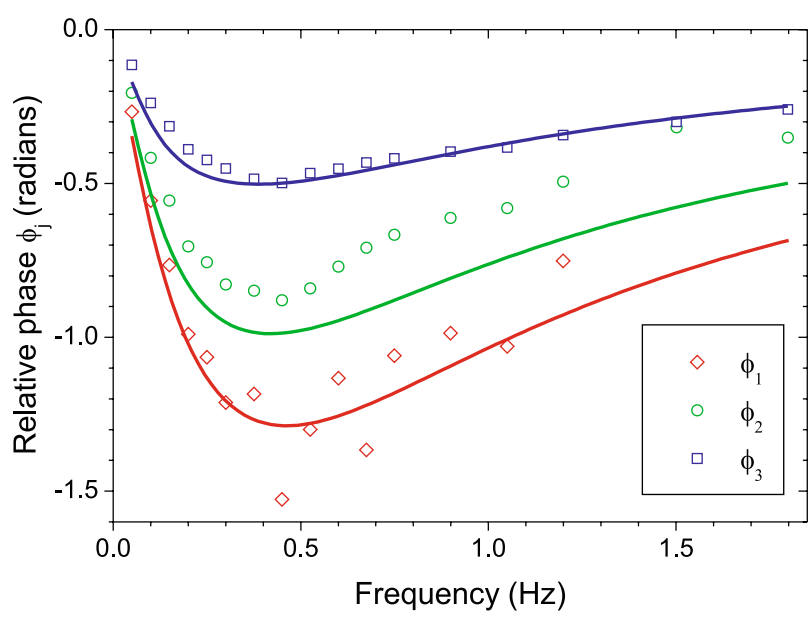

Fig. 2. (a) Measured frequency dependence of the relative amplitudes of the first three colloids (symbols) in the colloidal chain together with fitted analytical solutions (curves), see sect. 3. (b) Measured frequency dependence of the relative phases of the first three colloids (symbols) in the colloidal chain together with fitted analytical solutions (curves).

150 seconds. The recorded $x$-positions of the third colloidal particle for one typical experiment are presented in fig. 1b. It is clear that the colloidal particle in the chain oscillates harmonically with the driven frequency. Using discrete Fourier transformation, we have determined the amplitude and the phase of each colloidal particle in the chain. The frequency dependence of relative amplitudes and relative phases of the first three colloidal particles with regard to the fourth driven colloidal particle are presented in fig. 2. One can see that the amplitudes of the oscillations of the particles are strongly decreased by increasing the driving frequency due to the strong viscous damping of the liquid crystal. Colloidal particles, which are closer to the fourth driven colloid also oscillate with higher amplitudes and smaller phase shifts, as one can expect for a highly viscous medium.

Oscillations of colloidal particles in colloidal chains are determined by the mass of the particles, the effective interparticle binding, binding between the laser tweezers and the particle and the strong viscous damping of the parti- a)

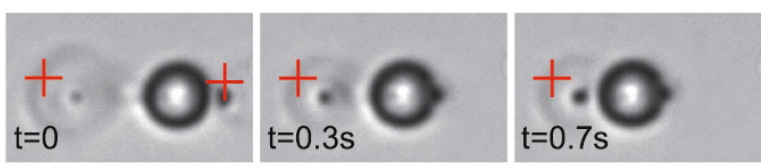

b)

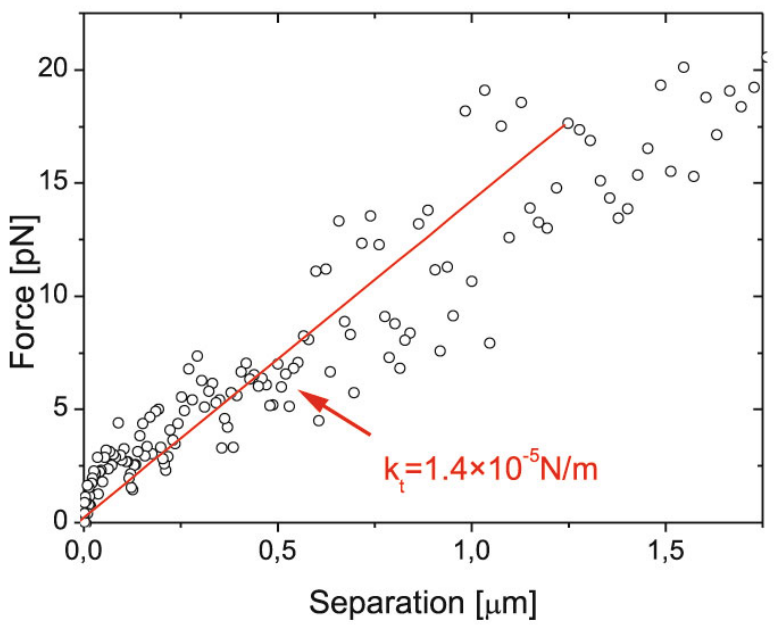

Fig. 3. Measurement of the spring constant $k_{t}$ between the optical trap and the colloidal particle with the Saturn ring. (a) Two optical traps are used at the beginning of the experiment. The first trap provides the attractive force between the laser tweezers focus and the particle and the second is used to hold the particle. The right trap is then switched off and the particle is attracted into the optical trap. (b) Optical force between the trap and the colloidal particle as a function of separation. The resulting spring constant of the optical trap is determined from the slope of the graph.

cles. We have therefore determined in three different experiments i) the effective viscosity of the particle $\gamma_{x}$, which determines the viscous drag force $F_{x}=\gamma_{x} \dot{x}$, ii) the effective spring constant of the entangled hyperbolic defect line which binds two neighboring particles, and iii) the effective spring constant between the laser tweezers and the particle. First the effective viscosity was determined by observing the thermal motion of the single Saturn-like particle [26]. By measuring the average displacement of the single particle, we have determined the effective viscosity in the horizontal direction $\gamma_{x}=6.6 \times 10^{-6} \mathrm{~kg} / \mathrm{s}$.

Second, we have measured the effective spring constant between the fixed trap of the laser tweezers and the particle $k_{t}$ by using two laser tweezers traps (fig. 3). The first optical trap was fixed at the one side of the Saturn ring, while the second trap grabbed the other side of the Saturn ring and stretched the Saturn ring. When the second optical trap was switched off, the released colloidal particle approached its equilibrium position. By measuring the time dependence of positions of that colloidal particle, the velocity of the colloidal particle $\dot{x}$ could be determined. Due to the high viscosity of the LC, the attractive force of the optical trap $k_{t} x$ is balanced with the Stokes viscous force $\gamma_{x} \dot{x}$. As shown in fig. $3 \mathrm{~b}$, the measured at- 

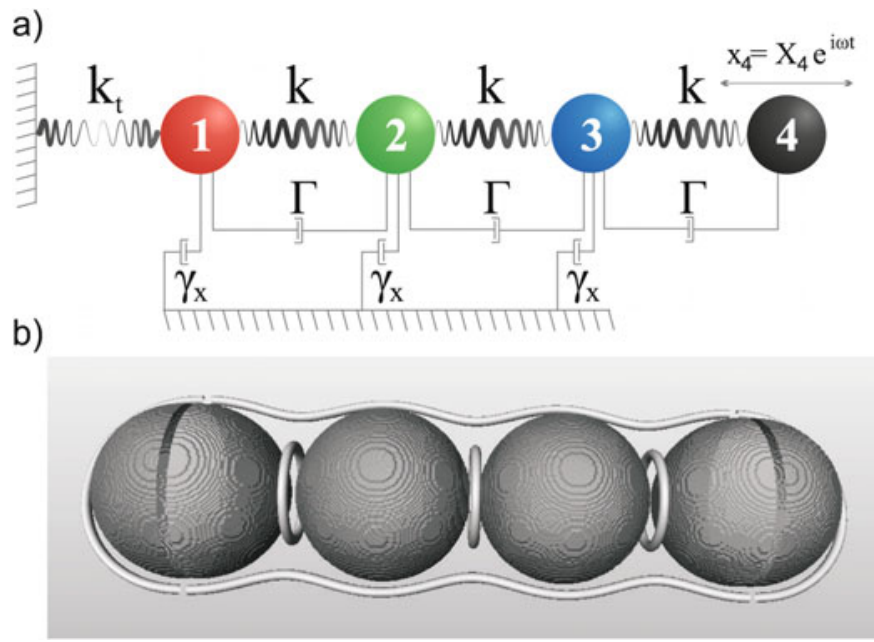

Fig. 4. (a) Bead-spring model for the vibrating entangled colloidal chain. The first particle interacts with the optical trap via the spring-like bond $k_{t}$. Note the spring-like bonds $k$ between the particles. $\gamma_{x}$ is the effective Stokes viscosity coefficient for the motion of particles and $\Gamma$ is the hydrodynamic interaction between neighboring particles. As in the experiment, particle No. 4 is harmonically driven into oscillation with amplitude $X_{4}$ and frequency $\omega$. (b) Hyperbolic defect colloidal chain, as calculated within the Landau-de Gennes approach [22]. The defects are visualized as isosurfaces with nematic order parameter $S=0.50$.

tractive force depends linearly on the separation between the optical trap and the colloidal particle and the effective spring constant $k_{t} \sim 1.4 \times 10^{-5} \mathrm{~N} / \mathrm{m}$ was determined.

Third, we have determined the effective spring constant $k$ of the entangled defect line and the hyperbolic defect in the experiment where an entangled pair of colloidal particles was stretched by moving two laser traps in the opposite directions to some predetermined position, and then the traps were switched off [22]. Again, similar to previous experiment, the attractive force of the entanglement, $k x$, was balanced by the viscous force $\frac{1}{2} \gamma_{x}\left(\dot{x}_{1}-\dot{x}_{2}\right)$. We found that the measured attractive force, binding a pair of entangled colloidal particles, depends linearly on the separation between the two particles. The effective spring constant of the hyperbolic defect line was $k \sim 2.3 \times 10^{-5} \mathrm{~N} / \mathrm{m}$. We can now compare the damping coefficient $\gamma_{x} / m \sim 7.2 \times 10^{6} \mathrm{~s}^{-1}$ with the natural angular frequency of the elastically bound colloids, $\sqrt{k / m} \sim 5.0 \times 10^{3} \mathrm{~s}^{-1}$, where $m \sim 9.2 \times 10^{-13} \mathrm{~kg}$ is the mass of the colloidal particle. Since the damping coefficient is much higher than the eigenfrequency of the oscillator, the oscillations of entangled nematic colloidal chains in LC are highly overdamped.

\section{Theory and discussion}

Forced vibrations of a colloidal chain, bound by entangled hyperbolic defects (fig. 4b), can be qualitatively described by an effective bead-spring model [27]. Three basic phenomena characterize the vibrations: i) the effective liquid crystal mediated inter-particle binding, ii) strong (Stokes) viscous damping of the movement of the particles, and iii) hydrodynamic coupling between neighboring particles. All three phenomena intertwine in the coupled amplitude and phase response of vibrated particles. Typically, at low frequencies (i.e. below $\sim 0.5 \mathrm{~Hz}$ ), the response of particles is determined primarily by the attractive inter-particle potential of the liquid crystal, whereas at higher frequencies $(\gtrsim 1 \mathrm{~Hz})$, the response is saturated and is governed by the hydrodynamic coupling between the particles.

Figure 4a shows the diagram of the effective beadspring model for a colloidal chain with $N=4$ particles, applied to the experimental configuration shown in fig. 1. The attractive liquid crystal interactions - the "LC bonds" - between sequent particles are approximated by the effective springs with elastic constant $k$. Analogously, the interaction of the first particle with the optical trap can be represented by a separate spring with elastic constant $k_{t}$. Dynamics of vibrating particles is deeply in the low-Reynolds-number regime $\left(R e \sim 10^{-6}\right)$, therefore Stokes drag force acting on the $j$-th particle is $F_{j}=\gamma_{x} \dot{x}_{j}$, where $\gamma_{x}$ is the effective viscosity coefficient and $\dot{x}_{j}$ is the velocity of the $j$-th particle. The hydrodynamic coupling between the particles is introduced via effective hydrodynamic forces and is assumed to be isotropic with a single friction coefficient $\Gamma[28]$. The elastic constants, the effective viscosity, and the friction coefficient, are used as phenomenological constants, whereas in a more general sense they are dependent on the inter-particle separation.

In the low-Reynolds-number regime and therefore neglecting inertia of colloidal particles, the dynamic equations of particles $j=1,2,3$ are written as

$$
\begin{aligned}
& 0=k\left(x_{2}-x_{1}\right)-\gamma_{x} \dot{x}_{1}+\Gamma\left(\dot{x}_{2}-\dot{x}_{1}\right)+k_{t} x_{1}, \\
& 0=k\left(x_{1}-2 x_{2}+x_{3}\right)-\gamma_{x} \dot{x}_{2}+\Gamma\left(\dot{x}_{1}-2 \dot{x}_{2}+\dot{x}_{3}\right), \\
& 0=k\left(x_{2}-2 x_{3}+x_{4}\right)-\gamma_{x} \dot{x}_{3}+\Gamma\left(\dot{x}_{2}-2 \dot{x}_{3}+\dot{x}_{4}\right) .
\end{aligned}
$$

Here, the motion of the particles is along the $x$ axis, with particle amplitudes $X_{j}$. In eqs. (1)-(3), the first terms characterize the attractive LC-mediated binding between the particles, the second terms represent viscous Stokes damping, and the third terms determine the hydrodynamic coupling between sequent particles. In eq. (1), the last term accounts for the interaction between the optical trap and the first particle. To mimic the experiment, the fourth particle is harmonically oscillated as $x_{4}=X_{4} \mathrm{e}^{i \omega t}$ with fixed amplitude and frequency.

We use the harmonic Ansatz for the vibrational amplitudes of particles $x_{j}=X_{j} \mathrm{e}^{i \omega t}$, and in this case eqs. (1)-(3) can be solved analytically, e.g., by standard functions in Mathematica 7.0. The following solutions for the complex particle amplitudes $X_{j}$ are obtained:

$$
\begin{aligned}
& X_{1}=\frac{-i A^{3}}{D} X_{4}, \\
& X_{2}=\frac{-A^{2} C}{D} X_{4}, \\
& X_{3}=\frac{A\left(i \gamma_{x} \omega\left(k_{t}-k\right)+\gamma_{x} \Gamma \omega^{2}-C^{2}+k_{t}^{2}\right)}{D} X_{4} .
\end{aligned}
$$


Here $A=\Gamma \omega-i k, B_{n}=\left(\gamma_{x}+n \Gamma\right) \omega, C=i B_{1}+k+$ $k_{t}$, and $D=A^{2}\left(i B_{2}+2 k\right)-\left(B_{1}-i k\right)\left(B_{3}-3 i k\right)\left(i B_{1}+\right.$ $\left.k+k_{t}\right)$ are the material parameters, dependent on the vibration frequency. Finally, to allow for comparison with experiments, relative amplitudes $\hat{X}_{j}$ and relative phases $\phi_{j}$ are introduced as $\hat{X}_{j}=\mathrm{e}^{i \phi_{j}} X_{j} / X_{4}$, where $\hat{X}_{j}, \phi_{j} \in \Re$.

The analytical solutions for the relative amplitudes $\hat{X}_{j}$ and relative phases $\phi_{j}$ were used to fit the experimental data (see fig. 2). The set of equations in eqs. (1)-(3) was however predetermined by one material parameter (e.g. the whole set can be divided by $k, \gamma_{x}$, or $\Gamma$ ), therefore only $k_{t}$ and two out of $\left(k, \gamma_{x}, \Gamma\right)$ could be extracted. To obtain the physical values of the modeling parameters, we thus first independently extracted the effective viscosity coefficient $\gamma_{x}=6.6 \times 10^{-6} \mathrm{~kg} / \mathrm{s}$ from the measured Brownian motion of a single particle.

The fits of the analytical solutions to the measured oscillation amplitudes and phases in fig. 2 correspond to the material parameters $k=1.41 \times 10^{-5} \mathrm{~N} / \mathrm{m}, k_{t}=$ $1.11 \times 10^{-5} \mathrm{~N} / \mathrm{m}$, and $\Gamma=2.8 \times 10^{-6} \mathrm{~kg} / \mathrm{s}$. Note that full experimental data of both relative amplitudes phases was simultaneously fitted for all three particles with the three material parameters. Good qualitative agreement between calculated (fitted) solutions and experimental data is observed in the full frequency range. Indeed, the material constants $k$ and $k_{t}$, extracted by fitting, qualitatively agree with those measured in the larger number of different experiments, $k=2.3(1 \pm 0.2) \times 10^{-5} \mathrm{~N} / \mathrm{m}$ and $k_{t}=1.4(1 \pm 0.2) \times 10^{-5} \mathrm{~N} / \mathrm{m}$. Here, the values of the elastic constant $k$ were measured on different entangled pairs of colloidal particles, which were first separated by using two laser tweezers traps and then released. On the other hand, the elastic constant $k_{t}$ was measured in different geometries, such as between the trap and a single colloidal particle with the Saturn ring, or between the trap and a pair of entangled particles. We have found that the overall accuracy of determining the elastic constants of the light trap is of the order of $\pm 20 \%$ and we cannot distinguish the differences in the effective trapping elastic constants for different experimental geometries.

To generalize the response of liquid crystal colloids to driven oscillations, a stability analysis of analytical solutions was performed. Liquid crystal inter-particle interaction, i.e. effectively the string constant $k$, affects the oscillation amplitudes and phases in the whole frequency interval. At lower frequencies $(\lesssim 1 \mathrm{~Hz})$, larger $k$ makes colloidal chain more rigid, whereas at higher frequencies the effect is reduced. Coupling of the optical trap to the colloidal structure (parameter $k_{t}$ ) affects substantially only the trapped particle, i.e. the first particle. On the other hand, Stokes friction has a strong impact on both amplitudes and phases in the whole frequency interval. If we increase the effective viscosity coefficient $\gamma_{x}$ by a factor of 2 , the saturated oscillation amplitudes at larger frequencies $(\gtrsim 1.5 \mathrm{~Hz}$ ) decrease by $4.2,2.5,1.6$ for particles 1 , 2,3 , respectively. However, if $\gamma_{x}$ is decreased by a factor of 2 , the colloidal chain becomes effectively more rigid, the particles start to oscillate in phase, and the saturated amplitudes at larger frequencies $(\gtrsim 1.5 \mathrm{~Hz})$ increase by
$3.2,2.1,1.4$ for particles $1,2,3$, respectively. The hydrodynamic coupling - the parameter $\Gamma$ - affects both the amplitudes and phases primarily at higher frequencies $(\gtrsim 0.5 \mathrm{~Hz}$ ), where larger $\Gamma$ stiffens the chain, similarly as a smaller $\gamma_{x}$. It is important to note that both oscillation amplitudes and relative phases indeed must be considered simultaneously for a correct characterization of colloidal oscillations. For example, even if neglecting the hydrodynamic coupling $(\Gamma=0)$, the agreement with experiments can be achieved for oscillation amplitudes, but not simultaneously for relative phases.

\section{Conclusion}

In this work, we have presented experimental and theoretical analysis of forced oscillations of an entangled chain of colloidal particles in the nematic liquid crystal $5 \mathrm{CB}$. The colloidal oscillations were driven by the strongly focused light of the laser tweezers and the response was measured by particle tracking of the recorded colloidal motion. As expected, driven oscillations are strongly damped by the large viscosity of the liquid crystal, which limits the mechanical response of colloidal structures to the frequency range below $\sim 1 \mathrm{~Hz}$ for micrometer-diameter colloidal particles. We have shown that collective colloidal motion can be well described using a rather simple model of elastically coupled particles in viscous media, where the hydrodynamic coupling has to be considered as well.

Authors would like to thank Andrej Vilfan for help with the effective bead-spring model. Funding from ARRS P1-0099 and Center of Excellence NAMASTE is acknowledged. MR acknowledges support from Marie Curie grant ACTOIDS.

Open Access This article is distributed under the terms of the Creative Commons Attribution Noncommercial License which permits any noncommercial use, distribution, and reproduction in any medium, provided the original author(s) and source are credited.

\section{References}

1. P. Poulin, H. Stark, T.C. Lubensky, D.A. Weitz, Science 275, 1770 (1997).

2. P. Poulin, D.A. Weitz, Phys. Rev. E 57, 626 (1998).

3. S. Ramaswamy, R. Nityananda, V.A. Raghunathan, J. Prost, Mol. Cryst. Liq. Cryst. 288, 175 (1996).

4. O.V. Kuksenok, R.W. Ruhwandl, S. Shiyanovskii, E.M. Terentjev, Phys. Rev. E 54, 5198 (1996).

5. P. Poulin, V. Cabuil, D.A. Weitz, Phys. Rev. Lett. 79, 4862 (1997).

6. T.C. Lubensky, D. Pettey, N. Currier, H. Stark, Phys. Rev. E 57, 610 (1998).

7. Y.Ch. Loudet, P. Barois, P. Poulin, Nature 407, 611 (2000).

8. S.P. Meeker, W.C.K. Poon, J. Crain, E.M. Terentjev, Phys. Rev. E 61, R6083 (2000). 
9. M. Yada, J. Yamamoto, H. Yokoyama, Langmuir 18, 7436 (2002)

10. O. Guzman, E.B. Kim, S. Grollau, N.L. Abbott, J.J. de Pablo, Phys. Rev. Lett. 91, 235507 (2003).

11. Jun-ichi Fukuda, H. Stark, M. Yoneya, H. Yokoyama, Phys. Rev. E 69, 041706 (2004).

12. I.I. Smalyukh, S. Chernyshuk, B.I. Lev, A.B. Nych, U. Ognysta, V.G. Nazarenko, O.D. Lavrentovich, Phys. Rev. Lett. 93, 117801 (2004).

13. I. Muševič, M. Škarabot, U. Tkalec, M. Ravnik, S. Žumer, Science 313, 954 (2006).

14. M. Škarabot, M. Ravnik, S. Žumer, U. Tkalec, I. Poberaj, D. Babič, I. Muševič, Phys. Rev. E 77, 061706 (2008).

15. H. Stark, Phys. Rep. 351, 387 (2001).

16. Jun-Ichi Hotta, M. Masuhara, Appl. Phys. Lett. 71, 2085 (1997).

17. S. Joudkazis, M. Iwashita, T. Takahashi, S. Matsuo, H. Misawa, Appl. Phys. Lett. 74, 3627 (1999).
18. M. Yada, J. Yamamoto, H. Yokoyama, Phys. Rev. Lett. 92, 185501 (2004).

19. T.A. Wood, H.F. Gleeson, M.R. Dickinson, A.J. Wright, Appl. Phys. Lett. 84, 4292 (2004).

20. I.I. Smalyukh, A.N. Kuzmin, A.V. Kachynsi, P.N. Prasad, O.D. Lavrentovich, Appl. Phys. Lett. 86, 021913 (2005).

21. I. Muševič et al., Phys. Rev. Lett. 93, 187801 (2004).

22. M. Ravnik et al., Phys. Rev. Lett. 99, 247801 (2007).

23. M. Škarabot et al., Phys. Rev. E 77, 031705 (2008).

24. J.C. Crocker, D.G. Grier, J. Colloid Interface Sci. 179, 298 (1996).

25. M. Škarabot et al., Phys. Rev. E 73, 021705 (2006).

26. J.C. Loudet, P. Hanusse, P. Poulin, Science 306, 1525 (2004).

27. M.R. Wilson, L.M. Stimson, J.M. Ilnytskyi, Z.E. Hughes, in Computer Simulations of Liquid Crystals and Polymers, edited by P. Pasini, C. Zannoni, S. Žumer (Kluwer Academic Publishers, the Netherlands, 2005).

28. S. Henderson et al., Phys. Rev. E 64, 061403 (2001). 